\title{
An Initial Experience and Usefulness of Porcine Acellular Dermal Matrix (PADM) Assisted Prepectoral Breast Implant Surgery: Case Series and Systematic Review
}

\section{Woo Jin Song}

Soonchunhyang University Hospital Seoul: Soonchunhyang University Hospital https://orcid.org/00000002-8957-3950

Hyung Bo Sim

BIO plastic surgery

Hyun Gyo Jeong

Soon Chun Hyang University Hospital: Soonchunhyang University Hospital

Sang Gue Kang ( $\sim$ sgkang@schmc.ac.kr)

Soonchunhyang University Hospital Seoul: Soonchunhyang University Hospital

\section{Research}

Keywords: Breast reconstruction, Breast augmentation, Acellular dermal matrix, Porcine, Capsular contracture

Posted Date: April 19th, 2021

DOI: https://doi.org/10.21203/rs.3.rs-420953/v1

License: (c) (1) This work is licensed under a Creative Commons Attribution 4.0 International License.

Read Full License 


\section{An Initial Experience and Usefulness of Porcine Acellular Dermal Matrix (PADM)}

Assisted Prepectoral Breast Implant Surgery: Case series and Systematic Review

Running title: Porcine acellular dermal matrix application in reconstructive and cosmetic breast surgery

Woo Jin Song', Hyung Bo Sim², Hyun Gyo Jeong³, Sang Gue Kang ${ }^{1 *}$

${ }^{1}$ Department of Plastic and Reconstructive Surgery, Soonchunhyang University College of Medicine, Seoul; 2BIO Plastic Surgery; ${ }^{3}$ Department of Plastic and Reconstructive Surgery, Soonchunhyang University College of Medicine, Gumi

Address Correspondence: Sang Gue Kang, M.D., Ph.D., Department of Plastic \& Reconstructive Surgery, Soonchunhyang University Seoul Hospital, Soonchunhyang University College of Medicine, 59, Daesagwan-ro, Yongsan-gu, Seoul, Korea, 04401, Tel: 82-2-709-9283/ Fax: 82-2-705-3687 / E-Mail: sgkang@schmc.ac.kr 


\begin{abstract}
Background:

We report our experience with performing prepectoral placement breast implant surgery using Supporix ${ }^{\mathrm{TM}}$ (HansBiomed, Korea), a porcine acellular dermal matrix (PADM), for cosmetic and reconstructive indications. The clinical efficiency, safety, and cost-effectiveness of PADM were also discussed.

\section{Methods:}

A single-center, retrospective study was designed from December 2017 to December 2019. The participants were Korean female patients who had PADM-assisted prepectoral breast implant surgery performed by two surgeons (S.G.K. \& H.B.S.). All complications were registered and analyzed. A systematic review and meta-analysis of the complication rates after PADM-assisted prepectoral breast implant surgery were conducted for comparison with other studies. Subgroup analysis was performed in three groups according to the operation type: the cosmetic breast surgery (CBS) group, the immediate implant-based breast reconstruction (IIBR) group, and the delayed implant-based breast reconstruction (DIBR) group.
\end{abstract}

\title{
Results:
}

A total of 20 breasts in 16 patients were included in our study with a median follow-up period of 8.25 months. In a systematic review, 20 publications with a total of 2,504 breasts in 1,921 females were quantitively analyzed. The overall complication rates in our study and other studies were $14 \%$ and $24 \%$ in total group analysis, $0 \%$ and $12 \%$ in the CBS group, $62 \%$ and $26 \%$ in the IIBR group, and $0 \%$ and $28 \%$ in the DIBR group, respectively.

\section{Conclusion:}

Our data support the effectiveness of PADM-assisted prepectoral breast implant surgery, which was comparable to other studies. PADM was effective for reducing seroma and hematoma in the revision CBS group and the DIBR group. In the IIBR group, it was helpful in preventing implant loss and explantation.

Keywords: Breast reconstruction, Breast augmentation, Acellular dermal matrix, Porcine, Capsular contracture 


\section{Background}

Recently, prepectoral implant-based breast reconstruction using an acellular dermal matrix (ADM) has increased [1-3]. Because of concerns regarding complications from a subpectoral approach, including animation deformity, arm-shoulder morbidity restriction, pain, chest tightness, muscle spasm, and increased recovery time, the implant site has been changed to a prepectoral site with the application of ADM [4]. In a prepectoral approach, the proper use of ADM is important because it can reduce the risk of implant-related complications such as capsular contracture, rippling, palpability, and visibility that can occur when prepectoral implant placement is performed alone $[5,6]$. Currently, half of all implant-based reconstructions are performed with the use of an ADM [7] because it improves the aesthetic outcome by better inframammary fold control, creating a larger new implant pocket in immediate breast reconstruction, and masking the surface irregularities in implant coverage $[8,9]$. However, prepectoral implant placement using ADM for full coverage of the implant requires a much larger amount of ADM than subpectoral implant placement, which uses ADM for covering the inferior pole. However, the access and use of human ADM (HADM) in prepectoral implant placement remain limited due to the more expensive cost than subpectoral implant placement. Instead of using HADM, porcine ADM (PADM) has been used in a variety of surgical fields including breast implant surgery after the publication of an experimental study evaluating the use of PADM for dura replacement (XenoDerm TM, Acelity, San Antonio, Tx) in 1999 [10-14]. One study reported the strong histologic similarity and biocompatibility of PADM equivalent to that of HADM, without showing inferiority in function [15]. Although PADM is more cost-effective than HADM, data on the use of PADM are limited [3], and it has not been used much in the field of cosmetic breast implant surgery.

We present a comparative study on the safety of PADM by presenting the experience and outcomes of PADM-assisted prepectoral breast implant surgery for reconstructive and cosmetic indications. The clinical efficiency was also discussed by analyzing the complication rates and safety of PADM through a systematic review and meta-analysis.

\section{Materials and Methods}

\section{Participant selection for case series}

Institutional Review Board approval and informed consent were obtained for the retrospective chart review of consecutive, PADM-assisted prepectoral breast implant surgery cases performed at a single academic medical center (IRB file No. SCHUH 2019-06-006-002). After a retrospective chart review of PADM-assisted prepectoral breast implant surgery using PADM (Supporix ${ }^{\mathrm{TM}}$, HansBiomed, Korea) 
between December 2017 and December 2019, an analysis was performed. The candidates for our study were revisionary cosmetic breast surgery patients with severe capsular contracture or foreign body injection, immediate implant-based breast reconstruction, and delayed implant-based breast reconstruction. The following clinical characteristics were recorded: age, body mass index (BMI), preoperative diagnosis, operation type, implant volume, laterality, operation time, the follow-up period, and complications. Complications such as animation deformity, bottoming out, palpability, visibility, infection, seroma, flap necrosis, and capsular contracture were investigated. In addition, surgical costs according to the operation time and cost of the ADM were discussed.

\section{Surgical procedures}

Before inserting the implant, we determined the implant volume and prepared the prepectoral pocket. The implant volume was determined by the volume of the excised mastectomy specimen (for reconstructive indication) or the patient's preference and contralateral volume (cosmetic indication). If the width of the pocket was much larger than that of the implant, the lateral part including the serratus anterior muscle was sewn to narrow the width for preventing implant migration. PADM $30 \times 20 \mathrm{~cm}$ in size (Supporix ${ }^{\mathrm{TM}}$, HansBiomed, Korea) and a cohesive round-shaped silicone gel-filled implant (Bellagel, SmoothFine ${ }^{\circledR}$, HansBiomed, Korea) were used for prepectoral implant placement with a ravioli technique [16]. Before implant insertion, the PADM was emulsified for 30 minutes. After making a stab incision on the PADM, the implant was completely wrapped with half-folded PADM. The surrounding area was sewn with interrupted Vicryl ${ }^{\circledR} 3 / 0$ sutures. The remnant area was removed (Figure 1). After confirming that the pectoralis major muscle (PMM) was intact, the implant was inserted above the PMM. ADM-fixation suturing was not performed. One silicone suction drain was inserted between the ADM cover and the skin. Double-layer wound closure was performed with Monosyn ${ }^{\circledR} 4 / 0$ interrupted sutures and Nylon ${ }^{\circledR} 5 / 0$ interrupted sutures for skin closure. The drains were left in place in all cases until the drainage was less than $20 \mathrm{~mL}$ in a 24-hour period. All patients received an individualized, treated postoperative dressing to prevent complications (Supplementary Material. Video 1).

\section{Study selection and data extraction}

A systematic review of available literature was carried out in May 2020 using PubMed. Preferred Reporting Items for Systematic Reviews and Meta-Analyses (PRISMA) guidelines were thoroughly followed for the literature search, selection, and reporting of the available evidence. A standardized data abstraction form was developed and utilized by the authors (Figure 2). We included references that described the use of PADM in cosmetic and reconstructive breast surgery. A literature search was 
performed in PubMed using the search string "Porcine" AND "Acellular Dermal Matrix" AND "Breast". The search was limited to articles in the English language and publication years from 2010 2020. Initially, only case reports were excluded. The titles and abstracts were screened to meet the following inclusion criteria: human studies, biologic material, prepectoral breast reconstruction, prepectoral cosmetic breast surgery, histological data, and complications. Publications of brief communications, correspondence, discussions, letters, and conference abstracts were excluded. The articles deemed relevant were subsequently read and examined in full to assess their eligibility. The reference lists of the included articles were also examined to identify additional relevant papers. All publications that met the inclusion criteria were deemed potentially eligible with respect to complication rates and the effectiveness of PADM in breast surgery. A search for unpublished literature was not performed. Two authors (W.J.S. \& S.G.K.) abstracted the data independently and settled any differences by discussion.

\section{Data Analysis}

All statistical analyses were performed using Rex, an excel-based statistical analysis software (Version 3.5.0.1 RexSoft. Inc., Seoul, Korea). To determine the 95\% confidence intervals (95\% CI) of PADM complications by the meta-analysis of single proportions, Freeman-Tukey Double arcsine transformation was used for the summary statistics. The study-specific prevalence estimates were then weighted by the inverse of their variance to compute the pooled prevalence and its 95\% CI. Randomeffects pooled estimates were calculated. To check for between-study variance, a version of the DerSimonian-Laird method was used [17]. To compare the complication rates between our study and the other studies, we performed an exact test of the probability of success of the Bernoulli experiment. To compare the effect of PADM between our study and the other studies through the meta-analysis of complications, a target group was set up as the total group of patients who underwent breast surgery using PADM. Subgroup analysis was performed for the three groups according to operation type: the cosmetic breast surgery (CBS) group, the immediate implant-based breast reconstruction (IIBR) group, and the delayed implant-based breast reconstruction (DIBR) group. All complications requiring intervention were registered and analyzed by dividing them into eight categories (overall complications, infections, skin necrosis, seroma/hematoma, implant loss/explantation, implant exposure, capsular contracture, and minor complications) according to the number and probability. The minor complications included partial nipple necrosis, red breast syndrome, contour deformity, delayed wound healing, and others. Publication bias was assessed using funnel plots and Rucker's 
arcsine-Thompson's (AS-Thompson's) test. A sensitivity analysis was also conducted to assess the influence of publication bias estimated by fitting a Copas selection model.

\section{Results}

\section{Efficacy of PADM for Prepectoral Breast Implant Surgery}

A literature search was performed following the PRISMA guidelines. Our search yielded 78 original articles (33 articles in PubMed and 45 articles in the Web of Science). A total of 14 duplicates were removed. The titles and abstracts of the remaining 64 articles were reviewed and 34 articles were excluded due to non-English articles, a lack of relevancy, or they only addressed surgical techniques or experiments. Finally, 30 articles were selected for full-text review. Four were eliminated after a full-text review. Hence, a total of 26 articles were included in this paper for qualitative analysis. Studies $(n=20)$ that clearly specified the numbers of breasts undergoing unilateral and bilateral procedures, the operation type, and complication rates were included in the meta-analysis (Figure 3). In a systematic review, a total of 2,504 breasts in 1,921 female patients were analyzed for complication rates after PADM-assisted prepectoral implant placement. The pooled incidence of overall complications was $24 \%$ (95\% CI: $17 \%-32 \%)$. Substantial heterogeneity $\left(\mathrm{I}^{2}=93.97 \% ; p<0.01\right)$ was detected in the study. Therefore, subgroup analysis by operation type was conducted. The overall complication rate was $12 \%$ in the CBS group, $28 \%$ in the DIBR group, and $26 \%$ in the IIBR group. As a result of detailed complication analysis, seroma and hematoma had the highest prevalence in the CBS group (4\%) and the IIBR group (10\%). However, skin necrosis was the most prevalent in the DIBR group (8\%). The results of our systematic literature review are presented in Table 1 and Figure 4 [18-37]. Publication bias analysis conducted with AS-Thompson's test showed asymmetry in the funnel plot with statistic 0.70, $p>0.05$. This result means that significant asymmetry was found, and the results derived from this meta-analysis should be considered cautiously. Fourteen of the twenty-four studies fell outside the $95 \%$ confidence intervals denoted by the diagonal dashed lines shown in the funnel plot (Figure 5, above left), which suggests possible heterogeneity and publication bias. The contour plot (Figure 5, above right) for the Copas selection model showing the logarithm of the PFT (Freeman-Tukey Double arcsine transformation) from the meta-anaylsis may be sensitive to the range of $(\gamma 0, \gamma 1)$ values. As the probability of publishing the study with the largest standard error decreases from $100 \%$ to $48 \%$ estimated PFT decreases from 0.43 to 0.52 (Figure 5, below left). The model indicates statistically not significant residual publication bias (i.e., p-value $>10 \%$ ) at the diminishing probability of publication (Figure 5, below right). Overall, adjusting for selection bias, the Copas selection model estimated 0.25 (95\% CI, 0.18-0.32), as did the random-effects model estimate of 0.25 (95\% CI, $0.18-0.33$ ). 


\section{Patient Outcomes}

PADM-assisted prepectoral implant placement was performed for a total of 20 breasts in 16 female patients (four bilateral: all cosmetic indications; 12 unilateral: 11 reconstructive, one cosmetic indication). For two breasts with capsular contracture, neosubpectoral pocket revision surgery was performed. For nine breasts with cosmetic indications, eight needing immediate implant-based reconstruction, and three needing delayed implant-based reconstruction, PADM-assisted prepectoral implant placement was performed by two surgeons. The mean age of the patients was 50.2 years, and the mean BMI was 23.2. All patients asked for an intact PMM and preferred prepectoral implant placement with complete PADM coverage (Ravioli technique). For those with cosmetic indications, two had bilateral capsular contracture, two had foreign body injection, and one had implant rupture. Two patients who had undergone repeated augmentation mammoplasty showed bilateral capsular contracture. In one patient, grade IV capsular contracture of both lower poles was shown, and the implant had already been removed. Due to repeated surgeries, scar tissue formation was observed between the PMM and parenchyma. After total capsulectomy and release of the scar contracture by the scoring envelope, the prepectoral pocket was prepared. In another patient who showed grade III contracture, anterior capsulectomy with neosubpectoral implant insertion was performed. Two patients who had undergone foreign body injection for breast augmentation received revisional augmentation mammoplasty after removal of the foreign body (one case) and subcutaneous mastectomy (one case). For those with reconstructive indications, eight patients underwent immediate reconstruction followed by unilateral nipple-sparing mastectomy (NSM) and three patients underwent delayed reconstruction followed by a lumpectomy, skin-sparing mastectomy (SSM), and contralateral augmentation mammoplasty for breast symmetry. The median follow-up period was 8.25 months (minimum: 3 months, maximum: 24 months). The average operation time was 115 minutes for cosmetic surgery, 55 minutes for immediate reconstruction, and 267 minutes for delayed reconstruction. Arm and shoulder mobility showed no restrictions. No patients complained of postoperative pain. No animation deformity was reported. The cosmetic results and the patients' satisfaction were excellent. The implant rims were not visible or palpable. The breasts were smooth except for one case of capsular contracture (grade I). Preoperative and postoperative immediate reconstruction, delayed reconstruction, and cosmetic indication images are shown in Figures 6 - 8. Five complications (two cases of seroma, one case of mild infection, one case of contour deformity, and one case of partial nipple necrosis) occurred only in the three patients with immediate reconstruction. No major complications such as total skin necrosis or severe infection occurred during the mean follow-up period of eight months. A 57-year-old patient (No. 6 patient) showed contour deformity of the left breast at the 1-year postoperative follow- 
up. The patient was suspected of having an implant rupture and seroma formation on the computed tomography (CT) and magnetic resonance imaging (MRI) findings. There was no rupture of the implant in the reoperation findings. However, the patient wanted implant removal due to anxiety. A 48-yearold patient (No. 8 patient) showed seroma and partial nipple necrosis, which was healed by conservative treatment. A 56-year-old patient (No. 11 patient) showed seroma and mild infection with fever at one month postoperatively. A salvage procedure was performed without implant removal. She showed a good recovery. All seromas were managed by clinic-based aspiration. All case summaries are shown in Table 2.

\section{Case 1 (cosmetic breast surgery, Figure 6)}

A 68-year-old patient (No.1 patient) with capsular contracture (grade IV) on both breasts had a history of a third revisional augmentation mammaplasty performed by another institution. Although implant removal was performed during the third revision, severe lower pole scarring with capsular contracture was observed. Surgery was approached through an inframammary fold incision. The intraoperative findings showed severe scar formation throughout the pectoralis muscle and breast parenchyma. After total capsulectomy including scar tissue excision, scoring was performed in the portion where the capsular contracture was sufficiently released. The fourth revisional augmentation mammoplasty was performed by inserting a PADM/implant construct (Supporix ${ }^{\mathrm{TM}} / 250 \mathrm{cc}$ Bellagel SmoothFine ${ }^{\circledR}$ on both breasts) into the prepectoral space without fixation sutures. The operation time was 140 minutes. No complications occurred during the follow-up period.

\section{Case 2 (immediate implant-based breast reconstruction, Figure 7)}

A 53-year-old patient (No. 7 patient) was diagnosed with breast cancer in the upper-outer quadrant of the left breast and nipple-sparing mastectomy was performed. The mastectomy specimen weighed 285 g. Immediate breast reconstruction for the left breast was planned. Surgery was approached through a 7-cm long inframammary fold incision. Immediate breast reconstruction was performed by inserting a PADM/implant construct (Supporix ${ }^{\mathrm{TM}} / 275 \mathrm{cc}$ Bellagel SmoothFine ${ }^{\circledR}$ ) into the prepectoral space without fixation sutures. The operation time was 85 minutes. No complications occurred during the follow-up period.

\section{$\square \quad$ Case 3 (delayed implant-based breast reconstruction, Figure 8)}

A 45-year-old patient (No. 14 patient) was previously diagnosed with breast cancer in the left breast and treated by lumpectomy in 2008 . The left breast was slightly flat compared to the right. Upper pole skin thinning was observed due to radiation therapy. Depressed scars were seen in the previous incision. 
The right breast was also small. Thus, augmentation mammaplasty for the right breast and delayed breast reconstruction for the left breast were planned. Surgery was approached through a $3.5-\mathrm{cm}-$ long inframammary fold incision. Delayed breast reconstruction was performed by inserting a PADM/implant construct (Supporix ${ }^{\mathrm{TM}} / 200 \mathrm{cc}$ Bellagel SmoothFine ${ }^{\circledR}$ ) into the prepectoral space of the left breast. Augmentation mammaplasty was also performed by inserting a PADM/implant construct (Supporix ${ }^{\mathrm{TM}} / 150 \mathrm{cc}$ Bellagel SmoothFine ${ }^{\circledR}$ ) into the prepectoral space of the right breast. Fat injection (35 cc) was performed to correct the depressed scar on the upper pole of the left breast. The operation time was 257 minutes. No complications occurred during the follow-up period.

\section{Comparative analysis between our study and other studies}

In the comparative analysis, the overall rate of complications in the total group cohort was $14 \%$ in our study and $24 \%$ in the other studies, showing no significant difference. In the subgroup analysis, the overall complication rates in the CBS group and the DIBR group were lower in our study (CBS: $0 \%$ vs. $12 \%$; DIBR: $0 \%$ vs. $28 \%$ ), but not significantly different between our study and the other studies. Although most complications were minor and did not require hospitalization or reoperation ( $25 \% \mathrm{vs}$. $2 \%, p=0.01)$, the overall complication rate in the IIBR group was significantly higher in our study $(62 \%$ vs. $26 \%, p=0.03$ ). In particular, no complications were observed in the CBS group or the DIBR group in our research. In the CBS group, the incidence of infection, skin necrosis, implant loss and explantation, implant exposure, and capsular contracture was $0 \%$ in both our study and the other studies. In the IIBR group and the DIBR group, the incidence of implant exposure and capsular contracture was also $0 \%$ in both our study and the other studies. All comparative analysis data are provided in Table 3.

\section{Discussion}

In breast surgery, implants have been broadly used in reconstruction following nipple-sparing mastectomy or cosmetic surgery. When using an implant, the surgeon should decide the position of the implant pocket. However, opinions on the optimal pocket for a breast implant remain divergent. In cosmetic surgery, implants can be placed in a prepectoral position or a subpectoral position depending upon various factors such as implant selection, tissue thickness, skin quality, subcutaneous fat tissue quality, breast tissue, and breast size [38]. Many surgeons opt for the prepectoral pocket approach, which has many advantages such as natural breast shape, avoiding animation deformity, ease of dissection, and reduced patient discomfort after surgery [2,39]. Subpectoral pocket placement is also 
widely performed to minimize the risk of implant visibility, palpability, rippling, and capsular contracture known to occur more often in a prepectoral pocket [40].

While there are options for prepectoral and subpectoral pockets in cosmetic surgery, there is a limitation in choosing the pocket in breast reconstruction. After a nipple-sparing mastectomy, the skin flaps are extremely thin. Therefore, the traditional methods of subpectoral or dual-plane coverage are still the most used [41]. However, PMM detachment can cause several problems, including severe pain, animation deformities, weakness in the shoulder and arm power, and changes in the anatomical location of the PMM after surgery [2,39].

As an alternative to this, immediate prepectoral reconstruction using ADM has increasingly been implemented [1-3,39]. This approach is simple, fast, and preserves the PMM. Thus, it does not have the disadvantages of subpectoral implant placement. Preserving the PMM was reported to play a major role not only in the simplicity of the operation but also in improving the quality of life (QoL) of patients [1]. Cattelani et al. reported that the impairment of upper limb function may persist over the long-term, and thus, adversely influence the QoL. They also showed that prepectoral breast reconstruction allowed patients to recover the complete function of the upper limb more quickly with more improved QoL than subpectoral procedures [3]. Our results also showed that animation deformity could affect the QoL in terms of aesthetic outcomes. Sbitany et al. reported that animation deformity was significantly lower in the prepectoral breast reconstruction group than in the subpectoral group.[42] In our study, no patients showed upper limb mobility restriction or animation deformity. This contributed to their satisfaction and QoL.

Capsular contracture is one of the notable implant-related complications of breast surgery. The etiology of contracture is multifactorial, involving factors such as the incision type, implant texture, anatomical location, bacterial contamination, drain, tobacco smoking, and antibiotic use [38,43,44]. Implant location is also believed to affect capsular contracture, which is more frequent in the prepectoral location than in the subpectoral location [2,6,38,40,43-45]. Egeberg et al. demonstrated a more than 2-fold increase in the risk of capsular contracture in a prepectoral location than in a subpectoral breast implant location [44]. Blount et al. reported that microorganisms located on the surface of the implant could alter the course of the inflammatory response as a major contributor to the development of capsular contracture. They believed that subpectoral placement would have lower capsular contracture rates due to the enhanced blood supply of the surrounding PMM, thus preventing infection [40].

To reduce the risk of capsular contracture in the prepectoral approach, surgeons have used ADM to 
completely cover the implant. It is thought that ADM may alter the reactive processes at the tissueimplant interface, the so-called contact zone, thus decreasing the development of contracture [41]. ADM can also be used for treating and preventing capsular contracture. When it is used as a treatment for grades III to IV capsular contracture, it has been reported that complete ADM coverage showed good results without recurrence at a follow-up of 9.2 months compared to partial ADM coverage with a recurrence rate of $6.3 \%$ [42]. In our study, four cases of capsular contracture without recurrence were treated.

Previously, the majority of studies and evidence based on the use of ADM in breast surgery were based on HADM. However, many studies using nonhuman ADMs derived from bovine pericardium, bovine dermis, or porcine dermis have been recently reported because they showed a similar capacity as HADM in various surgical fields, including dura repair, ventral hernia, chest wall reconstruction, and breast surgery $[2,10,11,13,15,20,23,28,46]$. Ball et al. performed a direct comparison of porcine and bovine ADMs (BADM) in implant-based immediate reconstruction and reported that using bovine ADM showed significantly higher rates of skin erythema and a trend toward higher complication rates [20]. PADM was also histologically similar to HADM without being functionally inferior to HADM [15]. For this reason, we used a prepectoral implant location for PADM for the first time in Korea.

The use of PADM has several advantages compared to HADM. First, PADM is more economical and easier to supply than HADM. Second, it can avoid ethical conflicts because it uses a non-human dermal matrix. Third, the incidence of complications associated with the use of PADM is not high compared to HADM. In terms of cost, Cattelani et al. reported that ADM-assisted prepectoral breast reconstruction had economic superiority not only to tissue expander (TE) procedures but also to the subpectoral implant location when considering the significantly lower rate of second surgery required for achieving symmetry [3]. Since the plane of breast implant placement in Korea does not make a difference in cost, even if it is covered by insurance, prepectoral implant placement using HADM for full implant coverage is extremely expensive. However, PADM is more economical. Assuming that the same size ADM is used for complete implant coverage, which is about $30 \times 20 \mathrm{~cm}$, the price of PADM (about $\$ 7,000$ ) is almost one-third that of HADM (about $\$ 24,300$ ). Spear et al. reported that the significant cost of PADM was compensated for by fewer revisions and aesthetically improved outcomes in the overall reconstruction [8]. In this case series, insurance coverage for ADM was applied only in the case of reconstructive indications, and insurance for cosmetic indications was not provided. This point could be a new option for revision cosmetic patients who are paying for the procedure without insurance coverage. And patients with reconstructive indications may benefit economically from fewer reoperations. In terms of complications, Berna et al. reported that there was no capsular contracture in 
a 4-year follow-up period after prepectoral breast reconstruction using PADM, Braxon ${ }^{\circledR}$ (Decomed Srl, Venice, Italy).[6] Reitsamer also reported that, although minor complications including partial nipple necrosis and hemorrhage can occur, the cosmetic results were excellent and the patients were fully satisfied after prepectoral implant placement using PADM coverage [2].

The results of the comparative study analyzing the effect of PADM showed that the postoperative overall complication rate in our study was $14 \%$, which was lower than that in other studies. In our study, complications did not occur in the CBS group or the DIBR group. In contrast, the incidence of complications was relatively high in the IIBR group in our study. This difference might have been exaggerated due to the small number of cases. There were no major complications such as implant loss or explantation or implant exposure requiring hospitalization or reoperation. Compared to the other studies, complications related to PADM such as implant exposure and capsular contracture did not occur in the total group. Infection, skin necrosis, and implant loss also did not occur in the CBS group. Thus, the use of PADM during breast surgery is expected to be helpful in this field. In a systematic review of the literature, high complication rates (prevalence of 0.5 or higher) after PADM surgery were observed in three studies related to IIBR. Dikman et al. performed a retrospective study on the use of Strattice in single-stage breast reconstruction and reported that the complication rate was rather high at $78 \%$ (seroma, $21 \%$; skin necrosis, $20 \%$ ). The reoperation rate was also high at $23 \%$. The obvious factor influencing complications was the patient's choice. Those who received radiation therapy and smokers were more likely to undergo reoperation. Other causes included the quality of the skin flap after mastectomy and the learning curve for the surgeon's ADM application [34]. Two other studies by Mazari et al. and Malata et al compared Strattice and SurgiMend in immediate implant-based breast reconstruction. The most common complication was also seroma in one study. In that study, the cause of seroma was reported to be the high percentage of axillary surgery. Other complications such as implant loss, ADM loss, and reoperation could be due to the learning curve [22]. In another study, the incidence of skin erythema and complications were significantly high possibly due to higher mastectomy breast weight in the Strattice group [23].

Several limitations of our study should be noted. First, our study included a small number of cases and a short-term follow-up for complications. The proportion of complications tended to increase. This might be because the number of cases in our study was small, and the complications were mostly concentrated in the IIBR group. Recruitment of large patient populations and long-term follow-up are needed for further analysis. Second, despite the fact that subgroup analysis was performed, there was substantial heterogeneity in the meta-analysis due to the clinical diversity in the studies included. This was the result of the limited selection of papers from which to obtain maximum information on 
complications and safety in breast implant surgery with PADM. The cause of such heterogeneity may also reflect the technological advances over time from 2012 to 2019 and that these studies were from several different countries, with different study designs and patient groups. Among several studies comparing HADM, PADM, and BADM, only PADM was extracted for analysis. In subgroup analysis, there were inevitably overlapping studies in the study design. For example, one study compared two or more groups of CBS, IIBR, and DIBR, and some were divided into one-stage and two-stage breast reconstruction in IIBR, that timing of reconstruction may have an some different effect on PADM. This requires further research. Third, radiotherapy, chemotherapy, and skin diseases that would be confused with inflammation, immune diseases, and the use of topical steroids were not included in the data analysis, so the possible effect of these factors on the safety outcomes was not identified.

\section{Conclusion}

Our literature review and cases support the effectiveness of PADM-assisted prepectoral breast implant surgery. To the best of our knowledge, this was the first case series report of PADM-assisted prepectoral breast implant surgery in Korea. Our single-center complication rates were comparable to those of other studies. The overall complication rate was relatively low. PADM-assisted prepectoral breast implant surgery was particularly effective in revision cosmetic breast surgery and delayed implant-based breast reconstruction. In immediate implant-based breast reconstruction, it was helpful in preventing implant loss, implant exposure, and capsular contracture. PADM-assisted prepectoral breast implant surgery for cosmetic and reconstructive indications had an economical benefit due to its lower resource usage compared to HADM. It also provided fewer ethical conflicts and lower complication rates compared to HADM and avoided the disadvantages of subpectoral placement by detaching the PMM. However, interpreting the results of the included studies in systematic review should be more cautious due to the influence of publication bias and substantial heterogeneity. By all that, when consulting patients on breast implant surgery, PADM-assisted prepectoral implant placement could be another option besides traditional technique if it is provided with appropriate patient selection and an improved learning curve. 


\section{Table \& Figure legends}

Table 1. Characteristics of the studies included in the systematic review of PADM-assisted breast implant surgery with information on complication rates in a total of 2,504 breasts of 1,921 patients

Table 2. Patient demographics, operative details, and outcomes for a total of 20 breasts of 16 patients

Table 3. Comparative analysis of complication rates after PADM-assisted prepectoral breast implant surgery in our study and other studies

Figure 1. Total porcine acellular dermal matrix (PADM) coverage of the implant: (a) After making a stab incision on the PADM, (b) the surrounding area was sewn by interrupted Vicryl ${ }^{\circledR} 3 / 0$ sutures, and the remnant pieces were removed. (c) PADM was wrapped around the implant before insertion of the PADM/implant construct.

Figure 2. Data abstraction form used in the research.

Figure 3. PRISMA diagram of study selection

Figure 4. Forest plot of the incidence of overall complications after PADM-assisted prepectoral breast implant surgery according to operation type.

Figure 5. Funnel plot and Copas selection modeling for publication bias.

Figure 6. Cosmetic indication (grade IV capsular contracture). Preoperative (Lt.) and 6-month postoperative images (Rt.) of a patient with grade IV capsular contracture. After total capsulectomy and release of the contracture from the scoring envelope, prepectoral breast implant placement with PADM was performed.

Figure 7. Immediate breast reconstruction. Preoperative (Lt.) and 6-month postoperative images (Rt.) of a patient who underwent immediate prepectoral breast reconstruction with PADM.

Figure 8. Delayed breast reconstruction. Preoperative (Lt.) and 6-month postoperative images (Rt.) of a patient who underwent delayed prepectoral breast reconstruction with PADM.

Supplementary information. Video 1 . Overall surgical technique of prepectoral breast implant surgery with PADM. 


\section{Acknowledgements}

This research was supported by Soonchunhyang University Research Fund.

Author Contributions: WJ wrote and reviewed the manuscript, conceived the figures, and performed a bibliographic search. HG reviewed the manuscript and participated in the bibliographic search. SG and HB contributed to the manuscript review and data analyses. SG coordinated the activity of all participants, revised the manuscript, and gave final approval. All authors have read and agreed to the published version of the manuscript.

Funding: This research was supported by the Soonchunhyang University Research Fund (Grant No. is not applicable). No specific grant from any funding agency in the commercial or not-for-profit sectors was received.

\section{Declarations}

\section{Ethics approval and consent to participate}

This study was approved by the Institutional Review Board of Soonchunhyang University Hospital, Seoul, Korea (IRB no. SCHUH 2019-06-006-002). It adhered to all relevant tenets of the Declaration of Helsinki. Written informed consent was obtained from all patients.

\section{Consent for publication}

All authors approved the final version of the manuscript and agreed to its submission for publication.

\section{Competing interests}

The authors declare that they have no competing interests. 


\section{References}

1. Sigalove, S.; Maxwell, G.P.; Sigalove, N.M.; Storm-Dickerson, T.L.; Pope, N.; Rice, J.; Gabriel, A. Prepectoral Implant-Based Breast Reconstruction: Rationale, Indications, and Preliminary Results. Plast Reconstr Surg 2017, 139, 287-294.

2. Reitsamer, R.; Peintinger, F. Prepectoral implant placement and complete coverage with porcine acellular dermal matrix: a new technique for direct-to-implant breast reconstruction after nipplesparing mastectomy. J Plast Reconstr Aesthet Surg 2015, 68, 162-167.

3. Cattelani, L.; Polotto, S.; Arcuri, M.F.; Pedrazzi, G.; Linguadoca, C.; Bonati, E. One-Step Prepectoral Breast Reconstruction With Dermal Matrix-Covered Implant Compared to Submuscular Implantation: Functional and Cost Evaluation. Clin Breast Cancer 2018, 18, e703-e711.

4. Gruber, R.P.; Kahn, R.A.; Lash, H.; Maser, M.R.; Apfelberg, D.B.; Laub, D.R. Breast reconstruction following mastectomy: a comparison of submuscular and subcutaneous techniques. Plast Reconstr Surg 1981, 67, 312-317.

5. Schmitz, M.; Bertram, M.; Kneser, U.; Keller, A.K.; Horch, R.E. Experimental total wrapping of breast implants with acellular dermal matrix: a preventive tool against capsular contracture in breast surgery? J Plast Reconstr Aesthet Surg 2013, 66, 1382-1389.

6. Berna, G.; Cawthorn, S.J. Long term follow-up on prepectoral ADM-assisted breast reconstruction: evidences after 4 years. European Journal of Plastic Surgery 2017, 40, 255-258.

7. Gurunluoglu, R.; Gurunluoglu, A.; Williams, S.A.; Tebockhorst, S. Current trends in breast reconstruction: survey of American Society of Plastic Surgeons 2010. Ann Plast Surg 2013, 70, 103110.

8. Spear, S.L.; Parikh, P.M.; Reisin, E.; Menon, N.G. Acellular dermis-assisted breast reconstruction. Aesthetic Plast Surg 2008, 32, 418-425.

9. Vardanian, A.J.; Clayton, J.L.; Roostaeian, J.; Shirvanian, V.; Da Lio, A.; Lipa, J.E.; Crisera, C.; Festekjian, J.H. Comparison of implant-based immediate breast reconstruction with and without acellular dermal matrix. Plast Reconstr Surg 2011, 128, 403e-410e.

10. Khalil, H.H.; Kalkat, M.; Malahias, M.N.; Rhobaye, S.; Ashour, T.; Djearaman, M.G.; Naidu, B. Chest Wall Reconstruction with Porcine Acellular Dermal Matrix (Strattice) and Autologous Tissue Transfer for High Risk Patients with Chest Wall Tumors. Plast Reconstr Surg Glob Open 2018, 6, e1703.

11. Skovsted Yde, S.; Brunbjerg, M.E.; Gudmundsdottir, G.; Bazys, M.; Heje, M.; Engberg Damsgaard, T. Dural repair using porcine ADM: two cases and a literature review. Case Reports Plast Surg Hand Surg 2017, 4, 5-8.

12. Rolle, L.; Falcone, M.; Ceruti, C.; Timpano, M.; Sedigh, O.; Ralph, D.J.; Kuehhas, F.; Oderda, M.; Preto, M.; Sibona, M.; et al. A prospective multicentric international study on the surgical outcomes and patients' satisfaction rates of the 'sliding' technique for end-stage Peyronie's disease with severe shortening of the penis and erectile dysfunction. BJU Int 2016, 117, 814-820.

13. Barmettler, A.; Heo, M. A Prospective, Randomized Comparison of Lower Eyelid Retraction 
Repair With Autologous Auricular Cartilage, Bovine Acellular Dermal Matrix (Surgimend), and Porcine Acellular Dermal Matrix (Enduragen) Spacer Grafts. Ophthalmic Plast Reconstr Surg 2018, 34, 266-273.

14. Chaplin, J.M.; Costantino, P.D.; Wolpoe, M.E.; Bederson, J.B.; Griffey, E.S.; Zhang, W.X. Use of an acellular dermal allograft for dural replacement: an experimental study. Neurosurgery 1999, 45, 320327.

15. Ge, L.; Zheng, S.; Wei, H. Comparison of histological structure and biocompatibility between human acellular dermal matrix (ADM) and porcine ADM. Burns 2009, 35, 46-50.

16. Liliav, B.; Patel, P.; Jacobson, A.K. Prepectoral Breast Reconstruction: A Technical Algorithm. Plast Reconstr Surg Glob Open 2019, 7, e2107.

17. Jackson, D.; Law, M.; Barrett, J.K.; Turner, R.; Higgins, J.P.; Salanti, G.; White, I.R. Extending DerSimonian and Laird's methodology to perform network meta-analyses with random inconsistency effects. Stat Med 2016, 35, 819-839.

18. Glasberg, S.B.; Light, D. AlloDerm and Strattice in breast reconstruction: a comparison and techniques for optimizing outcomes. Plast Reconstr Surg 2012, 129, 1223-1233.

19. Hille-Betz, U.; Kniebusch, N.; Wojcinski, S.; Henseler, H.; Heyl, V.; Ohlinger, R.; Paepke, S.; Klapdor, R.; Krause-Bergmann, B. Breast reconstruction and revision surgery for implantassociated breast deformities using porcine acellular dermal matrix: a multicenter study of 156 cases. Ann Surg Oncol 2015, 22, 1146-1152.

20. Lardi, A.M.; Ho-Asjoe, M.; Junge, K.; Farhadi, J. Capsular contracture in implant-based breast reconstruction-the effect of porcine acellular dermal matrix. Gland Surg 2017, 6, 49-56.

21. Onesti, M.G.; Maruccia, M.; Di Taranto, G.; Albano, A.; Soda, G.; Ballesio, L.; Scuderi, N. Clinical, histological, and ultrasound follow-up of breast reconstruction with one-stage muscle-sparing "wrap" technique: A single-center experience. J Plast Reconstr Aesthet Surg 2017, 70, 1527-1536.

22. Mazari, F.A.K.; Wattoo, G.M.; Kazzazi, N.H.; Kolar, K.M.; Olubowale, O.O.; Rogers, C.E.; Azmy, I.A. The Comparison of Strattice and SurgiMend in Acellular Dermal Matrix-Assisted, ImplantBased Immediate Breast Reconstruction. Plast Reconstr Surg 2018, 141, 283-293.

23. Ball, J.F.; Sheena, Y.; Tarek Saleh, D.M.; Forouhi, P.; Benyon, S.L.; Irwin, M.S.; Malata, C.M. A direct comparison of porcine (Strattice) and bovine (Surgimend) acellular dermal matrices in implantbased immediate breast reconstruction. J Plast Reconstr Aesthet Surg 2017, 70, 1076-1082.

24. Berna, G.; Cawthorn, S.J.; Papaccio, G.; Balestrieri, N. Evaluation of a novel breast reconstruction technique using the Braxon((R)) acellular dermal matrix: a new muscle-sparing breast reconstruction. ANZ J Surg 2017, 87, 493-498.

25. Paprottka, F.J.; Krezdorn, N.; Sorg, H.; Konneker, S.; Bontikous, S.; Robertson, I.; Schlett, C.L.; Dohse, N.K.; Hebebrand, D. Evaluation of Complication Rates after Breast Surgery Using Acellular Dermal Matrix: Median Follow-Up of Three Years. Plast Surg Int 2017, 2017, 1283735.

26. Kilchenmann, A.J.; Lardi, A.M.; Ho-Asjoe, M.; Junge, K.; Farhadi, J. An evaluation of resource utilisation of single stage porcine acellular dermal matrix assisted breast reconstruction: A comparative study. Breast 2014, 23, 876-882. 
27. Salzberg, C.A.; Dunavant, C.; Nocera, N. Immediate breast reconstruction using porcine acellular dermal matrix (Strattice): long-term outcomes and complications. J Plast Reconstr Aesthet Surg 2013, $66,323-328$.

28. Lardi, A.M.; Ho-Asjoe, M.; Mohanna, P.N.; Farhadi, J. Immediate breast reconstruction with acellular dermal matrix: factors affecting outcome. J Plast Reconstr Aesthet Surg 2014, 67, 1098-1105.

29. Fakim, B.; Highton, L.; Gandhi, A.; Johnson, R.; Murphy, J. Implant-based breast reconstruction with Artia tissue matrix. J Plast Reconstr Aesthet Surg 2019, 72, 1548-1554.

30. Spear, S.L.; Sinkin, J.C.; Al-Attar, A. Porcine acellular dermal matrix (strattice) in primary and revision cosmetic breast surgery. Plast Reconstr Surg 2013, 131, 1140-1148.

31. Mitchell, R.E. Porcine acellular dermis-assisted breast reconstruction: influence of adjuvant radiotherapy on complications and outcomes. Plast Reconstr Surg Glob Open 2013, 1, e77.

32. Ng, C.E.; Pieri, A.; Fasih, T. Porcine acellular dermis-based breast reconstruction: complications and outcomes following adjuvant radiotherapy. Eur J Plast Surg 2015, 38, 459-462.

33. Gardani, M.; Simonacci, F.; De Sario, G.; Cattadori, F.; Raposio, E.; Palli, D. Prepectoral breast reconstruction using the Braxon ${ }^{\circledR}$ porcine acellular dermal matrix: a retrospective study. European Journal of Plastic Surgery 2019, 42, 145-154.

34. Dikmans, R.E.; El Morabit, F.; Ottenhof, M.J.; Tuinder, S.M.; Twisk, J.W.; Moues, C.; Bouman, M.B.; Mullender, M.G. Single-stage breast reconstruction using Strattice: A retrospective study. J Plast Reconstr Aesthet Surg 2016, 69, 227-233.

35. Pozner, J.N.; White, J.B.; Newman, M.I. Use of porcine acellular dermal matrix in revisionary cosmetic breast augmentation. Aesthetic surgery journal 2013, 33, 681-690.

36. Himsl, I.; Drinovac, V.; Lenhard, M.; Stockl, D.; Weissenbacher, T.; Dian, D. The use of porcine acellular dermal matrix in silicone implant-based breast reconstruction. Arch Gynecol Obstet 2012, 286, 187-192.

37. Loo, Y.L.; Haider, S. The Use of Porcine Acellular Dermal Matrix in Single-stage, Implant-based Immediate Breast Reconstruction: A 2-center Retrospective Outcome Study. Plast Reconstr Surg Glob Open 2018, 6, e1895.

38. Adams, W.P., Jr. Capsular contracture: what is it? What causes it? How can it be prevented and managed? Clinics in plastic surgery 2009, 36, 119-126, vii.

39. Sbitany, H.; Piper, M.; Lentz, R. Prepectoral Breast Reconstruction: A Safe Alternative to Submuscular Prosthetic Reconstruction following Nipple-Sparing Mastectomy. Plast Reconstr Surg 2017, 140, 432-443.

40. Blount, A.L.; Martin, M.D.; Lineberry, K.D.; Kettaneh, N.; Alfonso, D.R. Capsular contracture rate in a low-risk population after primary augmentation mammaplasty. Aesthetic surgery journal 2013, $33,516-521$.

41. Headon, H.; Kasem, A.; Mokbel, K. Capsular Contracture after Breast Augmentation: An Update for Clinical Practice. Arch Plast Surg 2015, 42, 532-543.

42. Cheng, A.; Lakhiani, C.; Saint-Cyr, M. Treatment of capsular contracture using complete implant 
coverage by acellular dermal matrix: a novel technique. Plast Reconstr Surg 2013, 132, 519-529.

43. Jacobson, J.M.; Gatti, M.E.; Schaffner, A.D.; Hill, L.M.; Spear, S.L. Effect of incision choice on outcomes in primary breast augmentation. Aesthetic surgery journal 2012, 32, 456-462.

44. Egeberg, A.; Sorensen, J.A. The Impact of Breast Implant Location on the Risk of Capsular Contraction. Ann Plast Surg 2016, 77, 255-259.

45. McGuire, P.; Reisman, N.R.; Murphy, D.K. Risk Factor Analysis for Capsular Contracture, Malposition, and Late Seroma in Subjects Receiving Natrelle 410 Form-Stable Silicone Breast Implants. Plast Reconstr Surg 2017, 139, 1-9.

46. Boules, M.; Strong, A.T.; Corcelles, R.; Haskins, I.N.; Ilie, R.; Wathen, C.; Froylich, D.; Sharma, G.; Rodriguez, J.; Rosenblatt, S.; et al. Single-center ventral hernia repair with porcine dermis collagen implant. Surg Endosc 2018, 32, 1820-1827. 


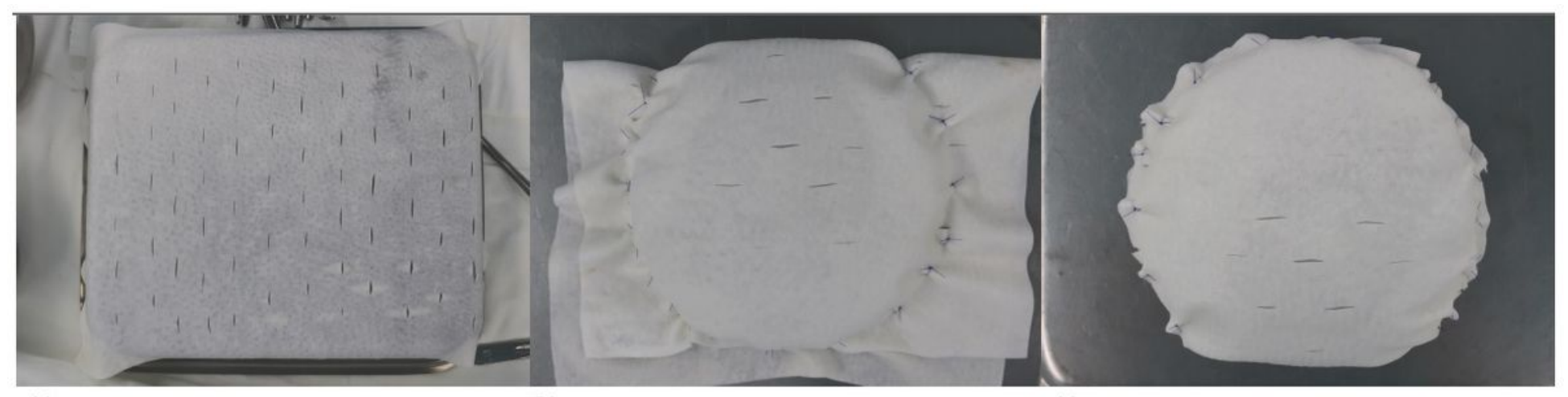

(a)

(b)

(c)

\section{Figure 1}

Total porcine acellular dermal matrix (PADM) coverage of the implant: (a) After making a stab incision on the PADM, (b) the surrounding area was sewn by interrupted Vicryl ${ }^{\circledR} 3 / 0$ sutures, and the remnant pieces were removed. (c) PADM was wrapped around the implant before insertion of the PADM/implant construct.

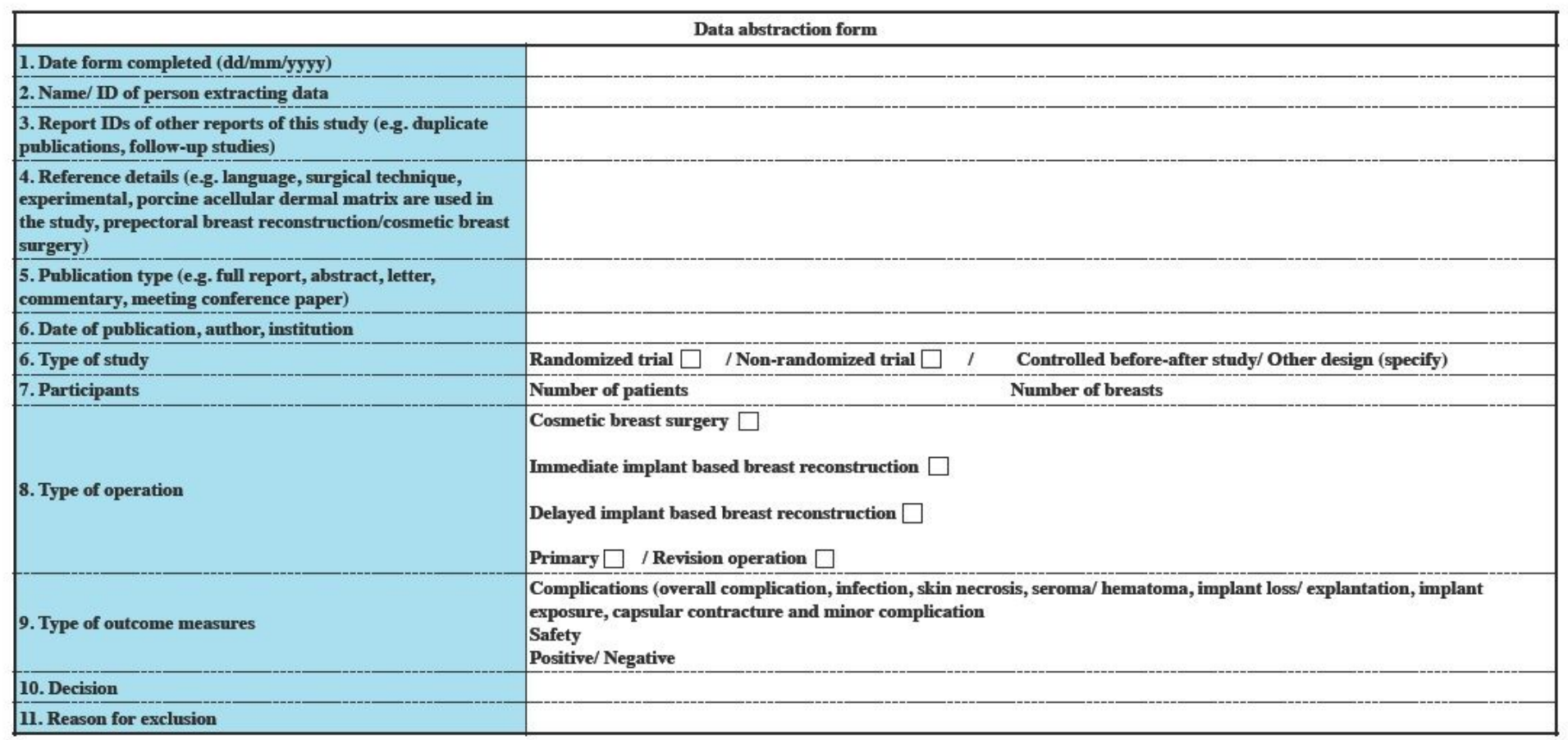

\section{Figure 2}

Data abstraction form used in the research. 

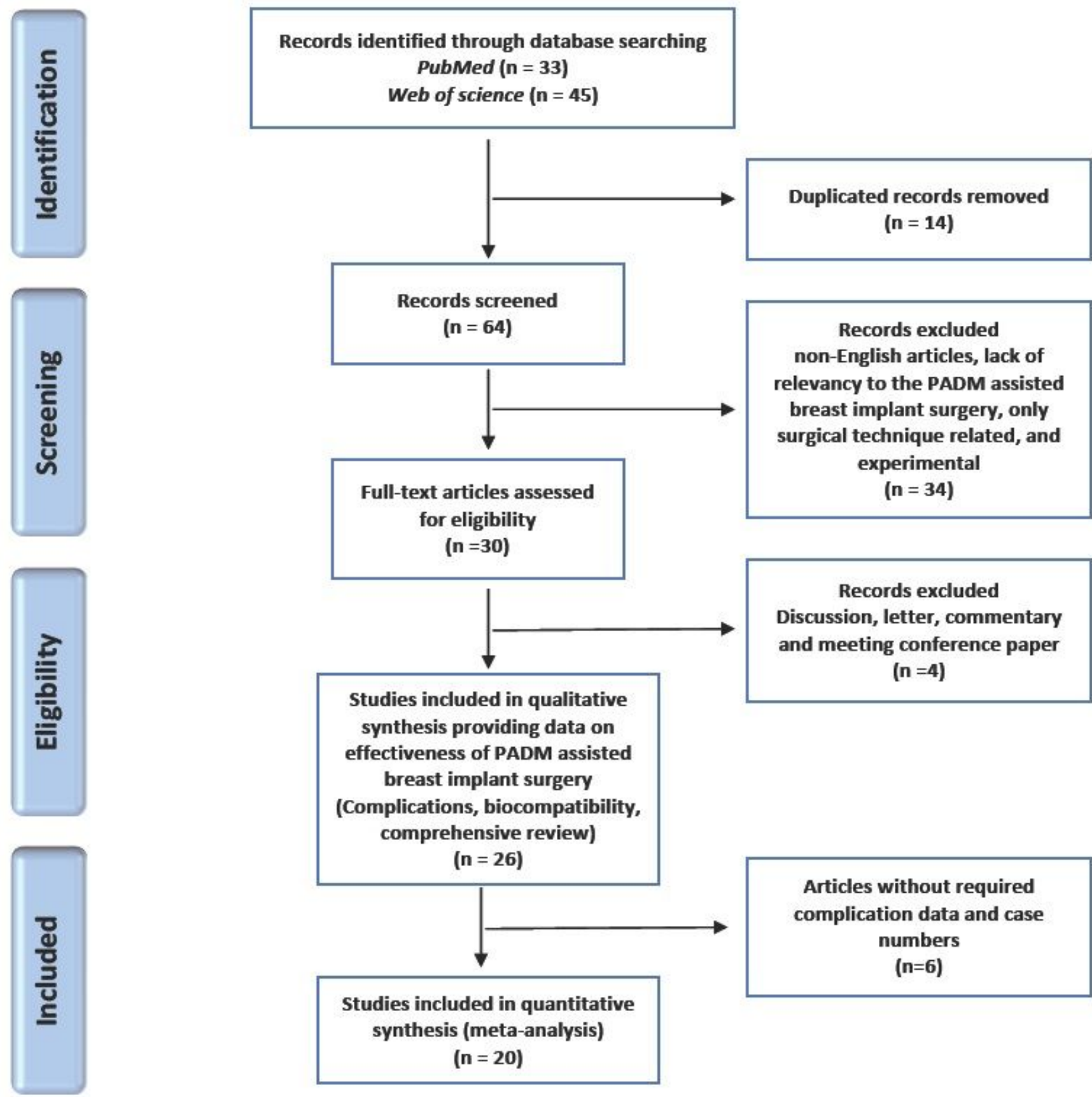

Studies included in qualitative synthesis providing data on effectiveness of PADM assisted breast implant surgery (Complications, biocompatibility, comprehensive review)

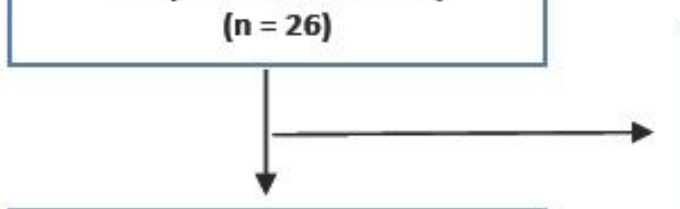

Articles without required complication data and case numbers ( $n=6)$

Studies included in quantitative synthesis (meta-analysis)

$$
\text { (n=20) }
$$

\section{Figure 3}


Subgroup: Cosmetic breast surgery

White et al. 2013

Spear et al. 2013

Hille-Betz et al. 2015

Paprottka et al. $2017^{\text {t* }}$

Random effect model

Heterogeneity: $\}^{2}=87.09 \%[68.99 \%, 94.62 \%], \tau^{2}=0.03, z^{2}=23.23(p \neq 0.01)$

Subgroup: Delayed implant-based breast reconstruction

Ng et al. $2015^{\text {** }}$

Hille-Betz et al. $2015^{* *}$

Paprottka et al. $2017^{*}$

Rantom effect model

Heterogeneity: $\gamma^{2}=64.21 \%[0.00 \% ; 89.75 \%], \tau^{2}=0.03, z_{2}^{2}=5.59(p=0.06)$

Subgroup: Immediate implant-based breast reconstruction

Looet al. 2018

Salzoerg et al: 2013

Kilchenmann et al. 2014

Himsl et al. 2012

Mazari et al. 2018

Glasberg et al. 2012

$\mathrm{Ng}$ et al. $2015^{*}$

Lardi et al. 2014

Mitchell et al. 2013

Hille Betre ot al. $2015^{*}$

Fakin et al. 2019

Maruccia et al. 2017

Malata et al. 2017

Lardi et al. 2017

Berra et al. 2017

Dikmans et al. 2016

Gardani et al. 2019

Random effect model

Heterogeneity $r^{2}=94.03 \%[91.83 \%, 95,64 \%], \tau^{2}=0.04, x_{16}^{2}=268(p<0.01)$

Random effect model

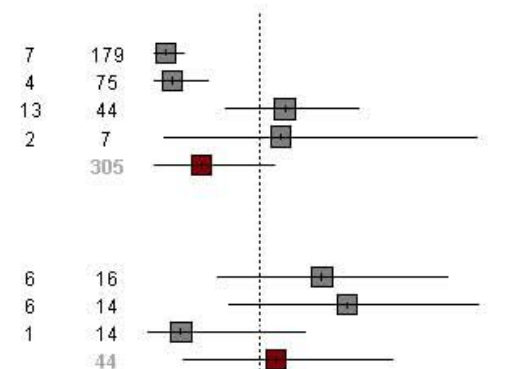

$0.04 \quad[0.02 ; 0.08]$ NA NA $\quad 4.68 \%$ [0.01;0.13] NA NA $4.48 \%$ $[0.17 ; 0.45]$ NA NA $\quad 4.26 \%$ $[0.04 ; 0.71]$ NA NA $2.69 \%$ $[0.02 ; 0.27]$

Heterogeneity: $f^{2}=93.97 \%[92.16 \% ; 95.37 \%], \tau^{2}=0.04, x_{23}^{2}=381.66(p<0.01)$

Test for subgroup differences (random): $x_{2}^{2}=2.82, \mathrm{df}=2(p=0.24)$

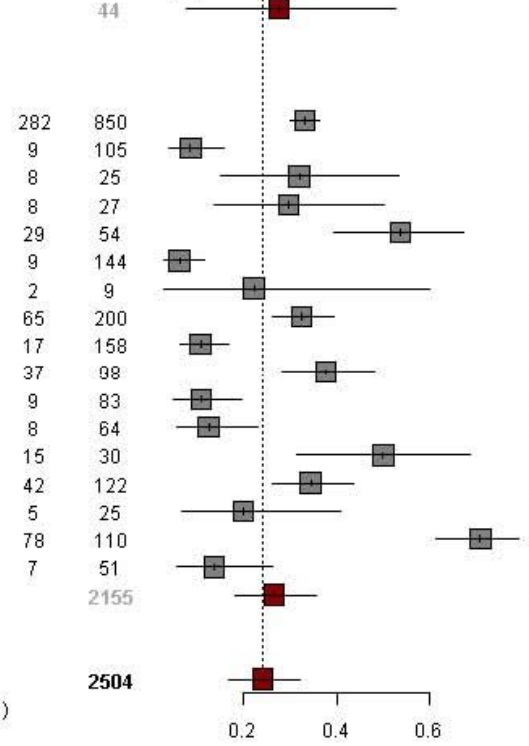

[0.15; 0.65] NA NA $3.54 \%$ $[0.18 ; 0.71]$ NA NA $3.42 \%$ $[0.00 ; 0.34]$ NA NA $\quad 3.42 \%$ $[0.08 ; 0.53]$

$10.38^{\circ}$

$[0.30 ; 0.36]$ NA NA $4.80 \%$ $[0.04 ; 0.16]$ NA NA $\quad 4.57 \%$ $[0.15 ; 0.54]$ NA NA $3.91 \%$ $[0.14 ; 0.50]$ NA NA $3.97 \%$ $[0.40 ; 0.67]$ NA NA $4.35 \%$ $[0.03 ; 0.12]$ NA NA $4.64 \%$ $[0.03 ; 0.60]$ NA NA $2.96 \%$

$[0.26 ; 0.39]$ NA NA $4.69 \%$ $[0.06 ; 0.17]$ NA NA $4.66 \%$ $[0.28 ; 0.48]$ N/ NA $\quad 4.56 \%$ $[0.05 ; 0.20]$ NA NA $4.51 \%$ $[0.06 ; 0.23]$ NA NA $4.42 \%$ $[0.31 ; 0.69]$ NA NA $4.04 \%$ $[0.26 ; 0.44]$ NA NA $4.61 \%$ [0.07; 0.41] NA NA $3.91 \%$ $[0.61 ; 0.79]$ NA NA $4.58 \%$ $[0.06 ; 0.26]$ NA NA $\quad 4.33 \%$ $[0.18 ; 0.36]=73.520$

$[0.17 ; 0.32] \quad 100.00^{\%}$

\section{Figure 4}

Forest plot of the incidence of overall complications after PADM-assisted prepectoral breast implant surgery according to operation type. 

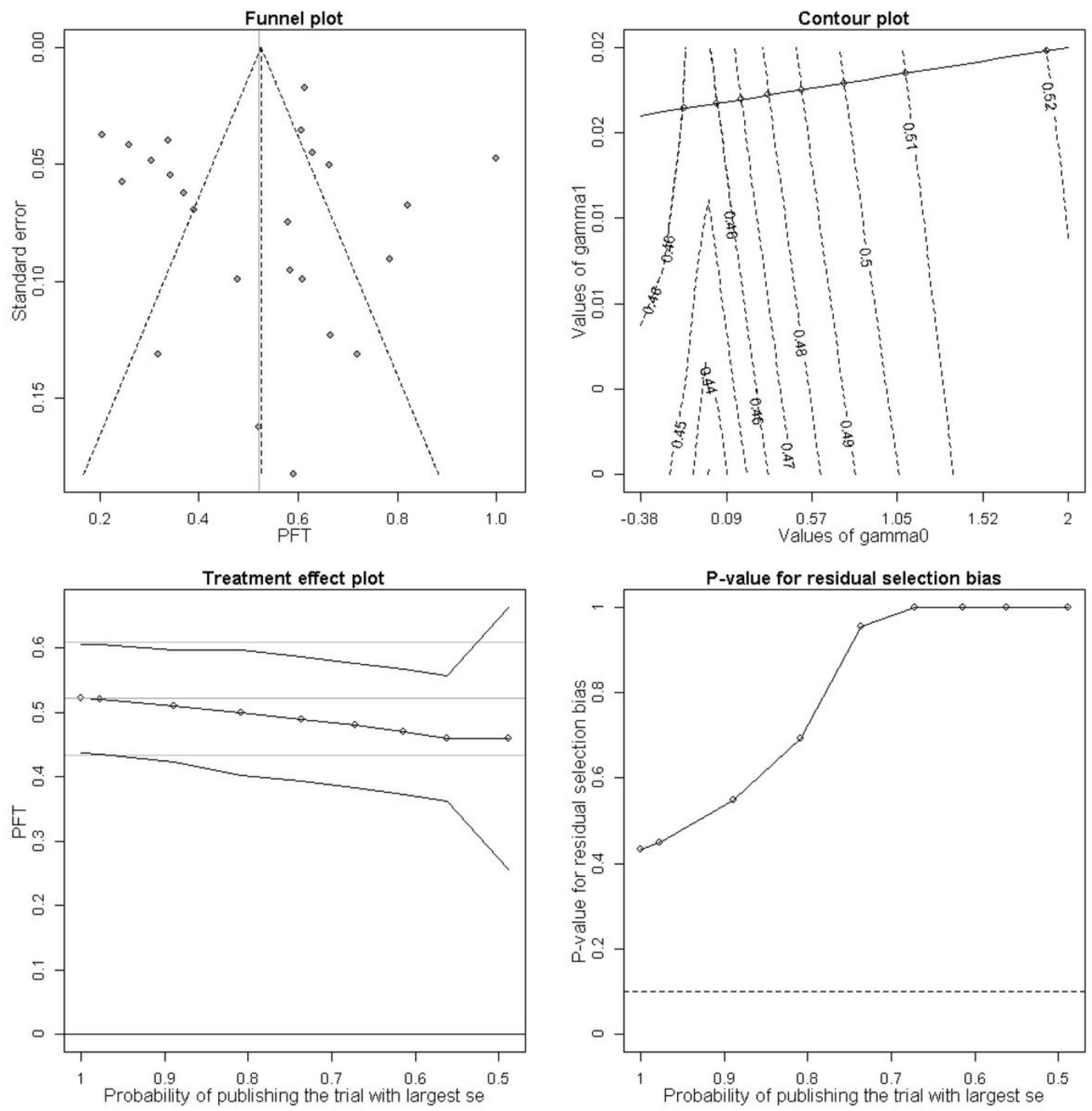

\section{Figure 5}

Funnel plot and Copas selection modeling for publication bias. 


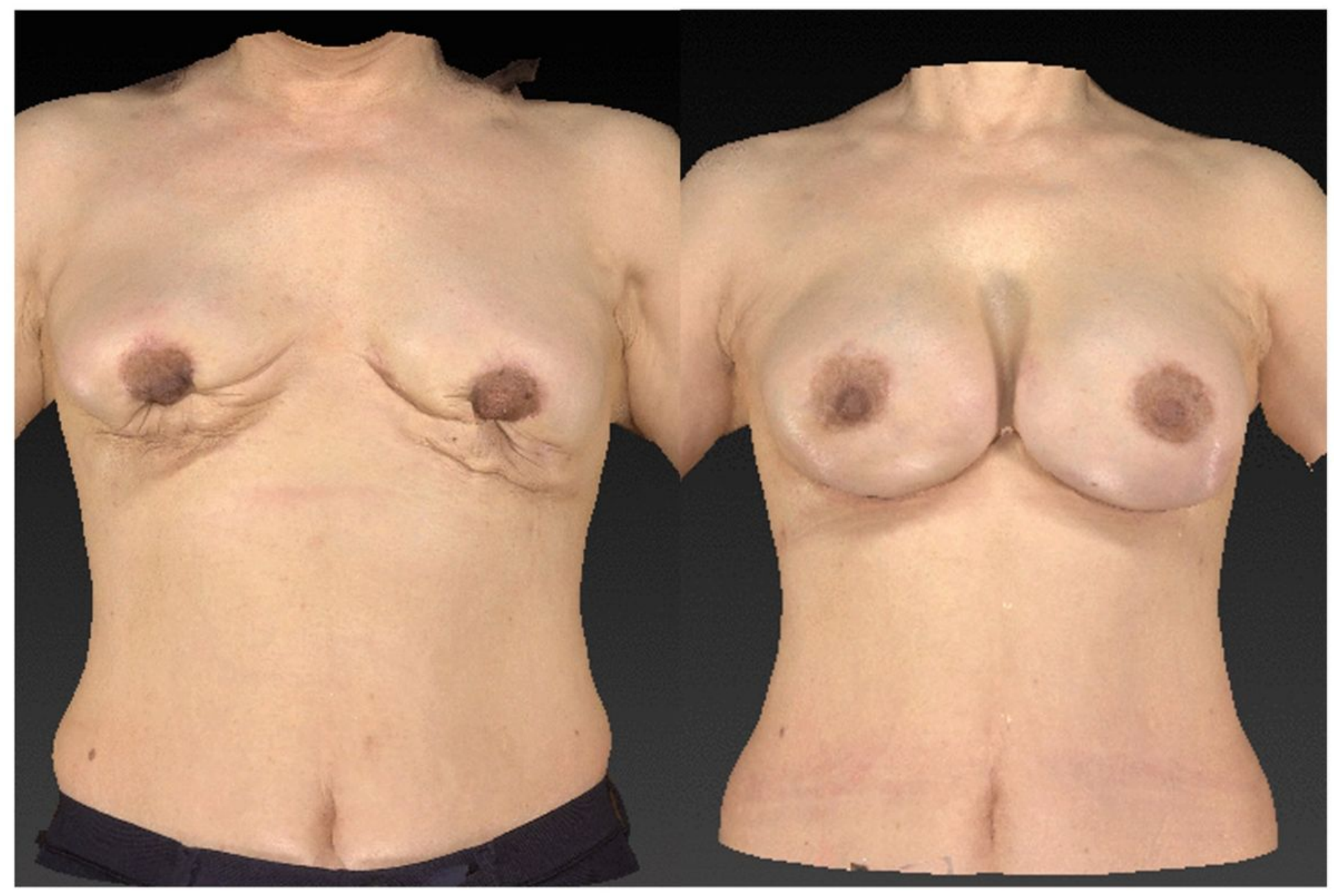

Figure 6

Cosmetic indication (grade IV capsular contracture). Preoperative (Lt.) and 6-month postoperative images (Rt.) of a patient with grade IV capsular contracture. After total capsulectomy and release of the contracture from the scoring envelope, prepectoral breast implant placement with PADM was performed. 


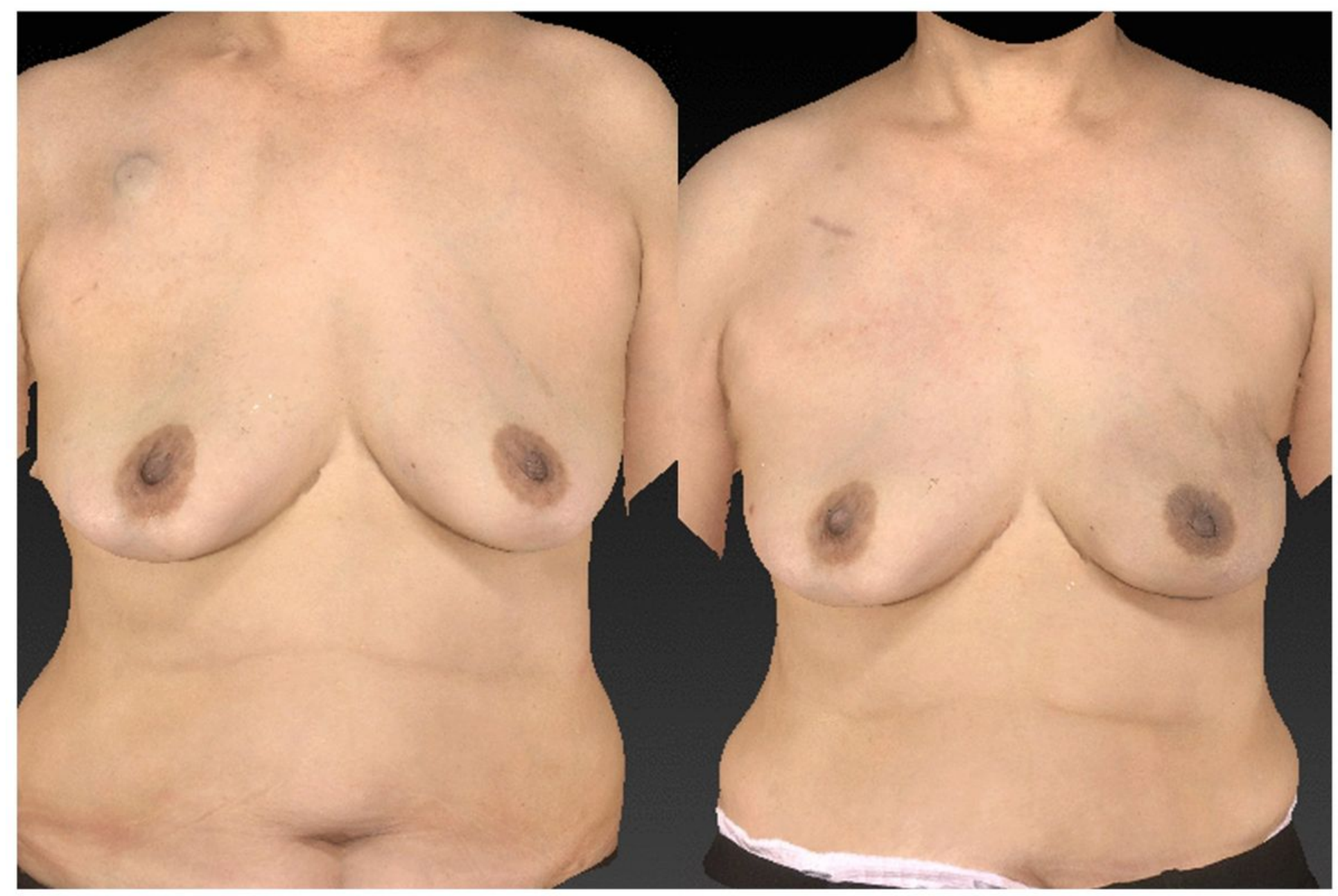

Figure 7

Immediate breast reconstruction. Preoperative (Lt.) and 6-month postoperative images (Rt.) of a patient who underwent immediate prepectoral breast reconstruction with PADM. 


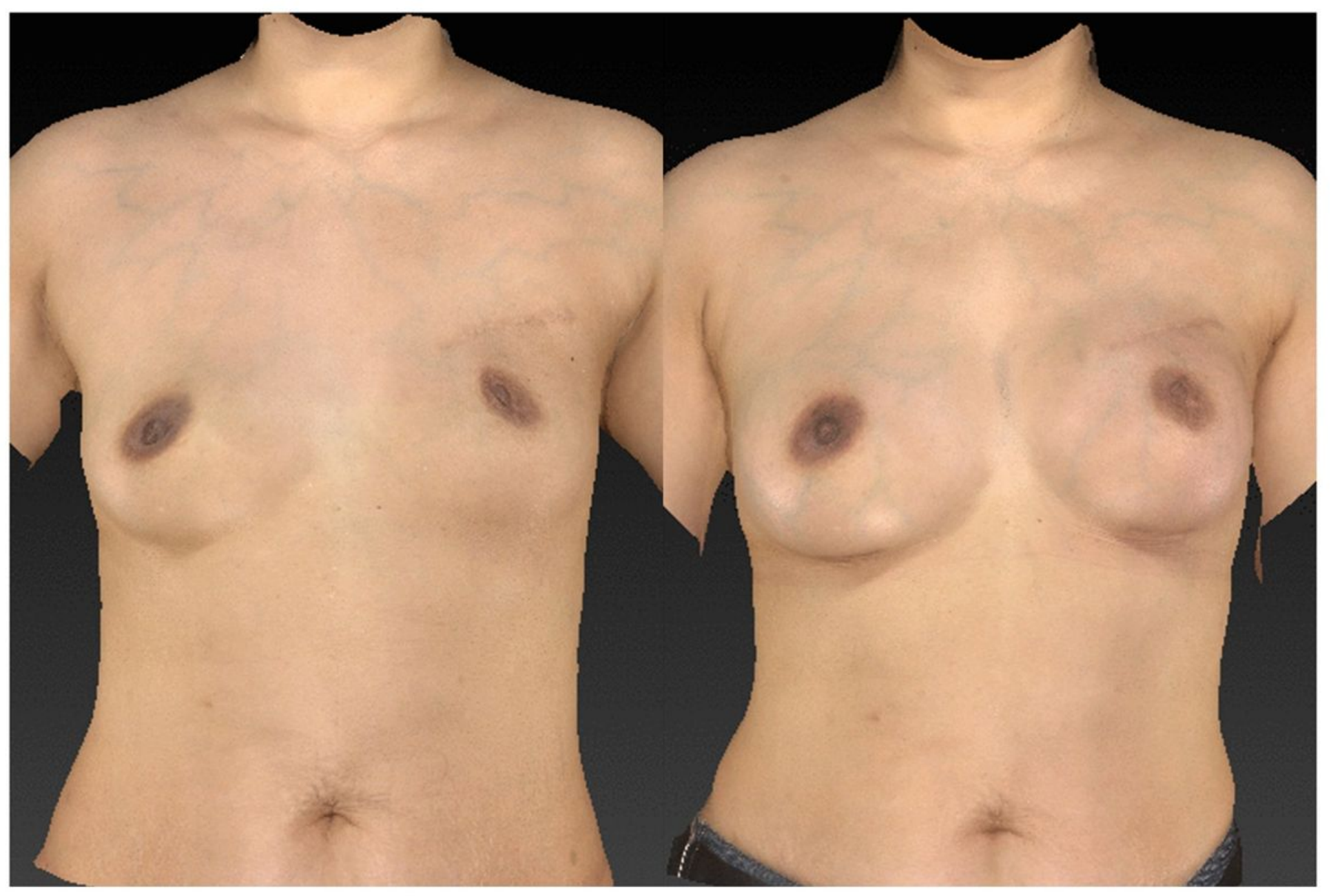

\section{Figure 8}

Delayed breast reconstruction. Preoperative (Lt.) and 6-month postoperative images (Rt.) of a patient who underwent delayed prepectoral breast reconstruction with PADM.

\section{Supplementary Files}

This is a list of supplementary files associated with this preprint. Click to download.

- E21031813Englishproofreadcertification.pdf

- PRISMA2009checklist.docx

- Supplementaldigitalcontent.mp4 